http://dx.doi.org/10.12775/szhf.2020.010

Alexei N. Krouglov

Russian State University for the Humanities, Moscow, Russian Federation

E-MAIL: AKROUGLOV@MAIL.RU

ORCID: 0000-0002-1152-1309

\title{
Russische Editionen und Übersetzungen Kants. Vorläufige Ergebnisse aufgrund der deutsch-russischen Kant-Ausgabe (1994-2019)*
}

2019 ist der letzte Band der deutsch-russischen Kant-Ausgabe erschienen, die insgesamt fünf Bände in sieben Büchern enthält ${ }^{1}$. Aus diesem Anlass sollen einige vorläufige Ergebnisse sowohl dieser als auch der vorherigen russischen Editionen Kants formuliert und die Probleme, die heute vor der russischen Kant-Forschung stehen, besprochen werden.

Der Beitrag wurde mit der Unterstützung der RSF (РHФ) vorbereitet (Projekt Nr. 2018-00243).

${ }^{1}$ Vgl. Marina Fedorovna Bykova, "Novoye slovo v mirovom kantovedenii. O dvuyazychnom izdanii sochineniy Immanuila Kanta na nemetskom i russkom yazykakh", Istoriko-filosofskiy ezhegodnik 2015 (2015): 362-380; Alexei N. Krouglov, Dieter Hüning, "Neuer Band der deutsch-russischen Kant-Ausgabe erschienen", Kant-Studien 110 (2019): 186-188. Die russischen Namen und Buchtitel werden im Haupttext nach dem für die deutsche Sprache üblichen RAK-System transliteriert; in den Fußnoten und in der Bibliographie dagegen nach dem in der englischen Sprache verbreiteten Chicago Manual of Style. 


\section{Die bisherigen russischen Editionen und Übersetzungen Kants: Errungenschaften, Mängel, bevorstehende Aufgaben}

Im Jahre 1814 beschrieb Vasilij Trofimovič Narežnyj (1780-1825) in seinem damals viel gelesenen Roman Der russische Gil Blas oder Die Abenteuer des Fürsten Gawrila Simonowitsch Tschistjakow eine Komödie, in der die Weisheit einem Kind namens Nikandr aus der Bibliothek „Gesamtausgaben Leibnizs und Kants" schenkt ${ }^{2}$. Dabei existierte 1814 nicht nur keine deutsche Gesamtausgabe Kants, sondern überhaupt keine nennenswerte Ausgabe von Kants Werken. Die erste richtige Kant-Ausgabe ist 1796-1798 auf Latein in der Übersetzung von Friedrich Gottlieb Born (1743-1807) erschienen, während den Grundstein für die deutschsprachigen Ausgaben Karl Rosenkranz (1805-1879) und Friedrich Wilhelm Schubert (1799-1869) im Jahre 1838 legten 4 .

Die erste russische Übersetzung ist noch zu den Lebzeiten des Königsberger Philosophen im Jahre 1803 erschienen - es handelt sich um die Grundlegung zur Metaphysik der Sitten ${ }^{5}$. Obwohl der Wunsch, eine Gesamtausgabe Kants zu besitzen, in der russischen Kultur schon am Anfang des 19. Jahrhunderts aufkam, wurde er lediglich Mitte des 20. Jahrhunderts mehr oder weniger realisiert. Die wichtigsten Schriften Kants jedoch waren schon vor der Oktoberrevolution 1917 ins Russische übersetzt worden, manche davon gar mehrfach ${ }^{6}$. Eine Sonderstellung genießt in diesem Zusammenhang die

${ }^{2}$ Vasiliy Trofimovich Narezhnyy, Rossiyskiy Zhilblaz, ili Pokhozhdeniya knyazya Gavrily Simonovicha Chistyakova. Roman v shesti chastyakh (Petrozavodsk: Kareliya, 1983), 143.

${ }^{3}$ Immanuelis Kantii opera ad philosophiam criticam, lat. vertit Friedrich Gottlob Born. Vol. I-IV (Leipzig: Schwickert, 1796-1798).

${ }^{4}$ Immanuel Kant, Sämmtliche Werke, ed. Karl Rosenkranz, Friedrich Wilhelm Schubert, Bd. 1-12 (Leipzig: Leopold Voss, 1838-1842). Wenigstens in der Tradition Arthur Wardas (1871-1929) ist diese Ausgabe ein neuer Anfangspunkt, vgl. Arthur Warda, Die Druckschriften Immanuel Kants (bis zum Jahre 1838) (Wiesbaden: Staadt, 1919).

${ }^{5}$ Kantovo osnovaniye dlya metafiziki nravov, transl. Yakov Andreyevich Ruban (Nikolayev: V tipografii Chernomorskago shturmanskago uchilishcha, 1803).

${ }^{6}$ Vgl. die Bibliographie bis 1994: Immanuil Kant. Bibliograficheskiy ukazatel literatury na russkom yazyke 1803-1994 gg., ed. Lyudmila Sergeyevna Davydova (Moskau: IFRAN, 1996). 
Kritik der reinen Vernunft ${ }^{7}$. Eine Gesamtausgabe fehlte aber nach wie vor, was in den 30er Jahren des 20. Jahrhunderts sichtbar wurde, denn 1929 begann das Marx-Engels-Institut eine mehrbändige russischsprachige Ausgabe der Werke Hegels zu publizieren. Zumindest waren 1933 zwei Bände mit den vorkritischen Schriften Kants geplant ${ }^{8}$, aber die politische Situation verhinderte deren Publikation. Nach dem Beginn des Zweiten Weltkrieges und kurz vor dem Beginn des Großen Vaterländischen Krieges, als bereits zehn Bände Hegels auf Russisch erschienen waren, wurde der zweite Band der vorkritischen Schriften Kants in der Übersetzung von Boris Aleksandrovič Focht (1875-1946) veröffentlicht ${ }^{9}$, den der Verlag schon 1936 vorbreitet hatte. Der erste Band ist dagegen nie erschienen. Allem Vermuten nach lag die Ursache dieses seltsamen Schicksals der vorkritischen Schriften Kants in den politischen Ereignissen um die zwei ersten sowjetischen Publikationen Kants,

${ }^{7}$ Bis heute sind 9 verschiedene Varianten erschienen: Immanuil Kant, Kritika chistago razuma, transl. Mikhail Ivanovich Vladislavlev (Sankt-Petersburg: Tipografiya N. Tiblena, 1867); Immanuil Kant, Kritika chistago razuma, transl. Nikita Matveyevich Sokolov (Sankt-Petersburg: Popov, 1896-1897); Immanuil Kant, Kritika chistago razuma, transl. Nikita Matveyevich Sokolov (Sankt-Petersburg: Popov, 1902); Immanuil Kant, Kritika chistago razuma, transl. Nikolay Onufriyevich Losskiy (Sankt-Petersburg: Tipografiya Stasyulevicha, 1907); Immanuil Kant, Kritika chistago razuma, transl. Nikolay Onufriyevich Losskiy (Petrograd: Tipografiya Stasyulevicha, 1915); Immanuil Kant, "Kritika chistogo razuma", transl. Nikolay Onufriyevich Losskiy, Tsolak Gevorkovich Arzakanyan, Moisey Isaakovich Itkin, in: Immanuil Kant, Sochineniya $v$ shesti tomakh, ed. Valentin Ferdinandovich Asmus, Arseniy Vladimirovich Gulyga, Teodor Ilich Oyzerman, Bd. 3 (Moskau: Mysl, 1964); Immanuil Kant, Kritika chistogo razuma, new transl. Nikolay Onufriyevich Losskiy, Tsolak Gevorkovich Arzakanyan, Moisey Isaakovich Itkin (Moskau: Mysl, 1994); Immanuil Kant, "Kritika chistogo razuma", transl. Nikolay Onufriyevich Losskiy, Tsolak Gevorkovich Arzakanyan, Moisey Isaakovich Itkin, Iskra Stepanovna Adreeva, in: Immanuil Kant, Sochineniya v vosmi tomakh, ed. Arseniy Vladimirovich Gulyga, Bd. 3 (Moskau: ChORO, 1994); Immanuil Kant, "Kritika chistogo razuma”, transl. Nikolay Onufriyevich Losskiy, Tsolak Gevorkovich Arzakanyan, Moisey Isaakovich Itkin, Nelli Vasilyevna Motroshilova, in: Immanuil Kant, Sochineniya na nemetskom i russkom yazykakh, ed. Nelli Vasilyevna Motroshilova, Burkhard Tuschling, Bd. 2.1, 2.2 (Moskau: Nauka, 2006). Vgl. auch Immanuil Kant, Kritika chistogo razuma, transl. Nikolay Onufriyevich Losskiy, mit den Varianten der Übersetzung ins Russische und in die anderen europäischen Sprachen, ed. Vladimir Aleksandrovich Zhuchkov (Moskau: Nauka, 1998).

${ }^{8}$ Vgl. Boris Yulyevich Slivker, "„Dokriticheskiy“ Kant”, Pod znamenem marksizma 4 (1933): 180.

${ }^{9}$ Immanuil Kant, Sochineniya 1747-1777: v dvukh tomakh, ed. Boris Yulyevich Slivker, transl. Boris Aleksandrovich Fokht, Bd. 2: 1759-1777 gg. (Moskau: Sotsekgiz, 1940). 
nämlich die der ersten und der zweiten Auflagen der Prolegomena (1934, 1937) ${ }^{10}$.

Die erste Ausgabe der Schriften Kants ist erst nach dem Krieg erschienen; sie wurde in den 60er Jahren des 20. Jahrhunderts von V. F. Asmus, T. I. Ojzerman und A. V. Gulyga ${ }^{11}$ herausgegeben. Das Erscheinen dieser sechsbändigen Kant-Ausgabe in sieben Büchern war ein wichtiges gesellschaftliches Ereignis, selbst wenn diese Ausgabe vor allem für Bibliotheken und Subskribenten bestimmt war und trotz des Umstandes, dass für sowjetische Verhältnisse nur wenige Exemplare, nämlich 17000 Stück, gedruckt wurden. Diese Kant-Ausgabe machte die meisten Druckschriften Kants zum ersten Mal für russische Leser zugänglich. Auch Ausschnitte aus dem Briefwechsel Kants sowie dem Opus postumum waren zum ersten Mal übersetzt worden. Allerdings war aus der Religion innerhalb der Grenzen der bloßen Vernunft, die schon 1917, noch vor der Oktoberrevolution komplett ins Russische übersetzt worden war ${ }^{12}$, lediglich der erste Aufsatz publiziert worden ${ }^{13}$. Der Verlag „Mysl““ erklärte seine Absicht, die Kant-Ausgabe zu veröffentlichen, folgendermaßen: „Immanuel Kant ist der Begründer der deutschen klassischen Philosophie, die eine der theoretischen Quellen des Marxismus ist. Ohne das Studium der wichtigsten Schriften dieses Denkers lassen sich die Geschichte der Dialektik und die Rolle der klassischen deutschen Philosophie in deren Entwicklung nicht verstehen. Die Kenntnis der Werke Kants ist auch für die Kritik der zeitgenössischen bourgeoisen Philosophie wichtig, deren viele Richtungen auf die eine oder andere Weise auf die reaktionären Seiten des Kantianismus zurückgehen ${ }^{\text {“14 }}$. Der Verfasser der Einleitung

${ }^{10}$ Immanuil Kant, Prolegomeny, transl. Vladimir Sergeyevich Solovyev, Boris Aleksandrovich Fokht, ed. Artashes Khorenovich Saradzhev (Moskau: OGIZ, 1934); Immanuil Kant, Prolegomeny ko vsyakoy budushchey metafizike, mogushchey vozniknut v kachestve nauki, transl. Vladimir Sergeyevich Solovyev, Boris Aleksandrovich Fokht, Mikhail Aleksandrovich Dynnik (Moskau: Sotsekgiz, 1937).

${ }^{11}$ Immanuil Kant, Sochineniya $v$ shesti tomakh, ed. Valentin Ferdinandovich Asmus, Arseniy Vladimirovich Gulyga, Teodor Ilich Oyzerman (Moskau: Mysl, 1963-1966).

${ }^{12}$ Immanuil Kant, Religiya $v$ predelakh tolko razuma, transl. Nikita Matveyevich Sokolov (Sankt-Petersburg: Izdaniye Yakovenko, 1908).

${ }^{13}$ Immanuil Kant, "Ob iznachalno zlom v chelovecheskoy prirode", in: Immanuil Kant, Sochineniya v shesti tomakh, Bd. 4(2), ed. Valentin Ferdinandovich Asmus (Moskau: Mysl, 1965).

14 "Ot izdatelstva", in: Immanuil Kant, Sochineniya $v$ shesti tomakh, Bd. 1, ed. Arseniy Vladimirovich Gulyga (Moskau: Mysl, 1963), 5. 
Immanuel Kant - der Begründer der deutschen klassischen Philosophie, der berühmte sowjetische Philosoph Theodor Il'ič Ojzerman (1914-2017), fügte hinzu, dass die Übersetzungen „der früher publizierten Schriften Kants nicht ganz befriedigend sind, da diese Übersetzungen, die von den Anhängern der idealistischen Philosophie geschaffen worden waren, in einigen Fällen durch deren eigene Vorstellungen geprägt sind und dadurch die Gedanken Kants verdrehen ${ }^{\text {"15. }}$. Gemeint waren offensichtlich die Übersetzungen von Nikolaj Onufrievič Losskj (1870-1965), Nikolaj Matveevič Sokolov (1860-1908), Vladimir Sergeevič Solov'ev (1853-1900) u.a. Ojzerman brachte die Hoffnung zum Ausdruck, dass die neuen Übersetzungen sowie die neue KantAusgabe selbst im Ganzen „den Grundanforderungen der marxistisch-leninistischen geschichtsphilosophischen Wissenschaft entsprechen werden"16. Der Versuch, die älteren Übersetzungen gemäß der marxistisch-leninistischen Philosophie zu korrigieren, führte jedoch zu manchen Mängeln dieser Ausgabe. So brachten die Korrekturen, die verschiedene Kantforscher an der Übersetzung der Kritik der reinen Vernunft von Losskij im dritten Band und an der Übersetzung der Prolegomena von Solov'ev im vierten Band vornahmen zahlreiche Inkonsequenzen in die Ausgabe. Einige Änderungen waren schlechter als die Varianten, die etwa Solov'ev vorgeschlagen hatte. Sehr gelitten hat die Grundlegung zur Metaphysik der Sitten: Selbst der Titel dieser Schrift war irrtümlich korrigiert worden ${ }^{17}$. Aber bei allen Mankos war die mit einem sehr guten wissenschaftlichen Apparat ausgerüstete Kant-Ausgabe der 60er Jahre eine wichtige Quelle für einige Generationen russischer Leser und ist durchaus als verdienstvoll einzuschätzen.

Anfang der 90er Jahre des 20. Jahrhunderts war die Kant-Ausgabe der 60er Jahre eine bibliographische Seltenheit. Zum Teil wurde dieses Defizit 1994 durch eine neue Ausgabe in acht Bänden beseitigt, die der Germanist und Philosophiehistoriker Arsenij Vladimirovič Gulyga (1921-1996) herausgab; sie wurde dem 200. Jubiläum der Wahl Kants zum Mitglied der Russischen

15 Teodor Ilich Oyzerman, "Immanuil Kant - rodonachalnik klassicheskoy nemetskoy filosofii”, in: Immanuil Kant, Sochineniya v shesti tomakh, Bd. 1, 49.

${ }^{16}$ Oyzerman, "Immanuil Kant - rodonachalnik klassicheskoy nemetskoy filosofii", 49.

${ }^{17}$ Immanuil Kant, "Osnovy metafiziki nravstvennosti", in: Immanuil Kant, Sochineniya $v$ shesti tomakh, Bd. 4(1), ed. Valentin Ferdinandovich Asmus (Moskau: Mysl', 1965). In einer Rückübertragung ins Deutsche heißt es so etwa „Grundlagen der Metaphysik der Sittlichkeit“. 
Akademie der Wissenschaften (1794) gewidmet ${ }^{18}$. Neben den Schriften Kants, die in der vorherigen Ausgabe bereits publiziert waren, wurden hier auch einige neue Texte veröffentlicht, die in der Zeit von 1966 bis 1993 ins Russische übersetzt worden waren ${ }^{19}$. Dennoch handelte es sich keinesfalls um eine mechanische Wiedergabe bereits vorhandener Übersetzungen. In der Einleitung des Herausgebers ist kein Wort über die Prinzipien der neuen Kant-Ausgabe zu finden ${ }^{20}$, aber eine kurze Annotation des Verlags lautet: „Die Jubiläumsausgabe unterscheidet sich von der vorherigen durch ihre Fülle: Hier wurde praktisch alles Wesentliche gesammelt, was auf Russisch publiziert worden ist. Der zweite Unterschied ist die Qualität der Übersetzungen. Die klassischen Übersetzungen von N. Losskij, V. Solov'ev, P. Florenskij sind, so weit es möglich war, wiederhergestellt. Auch die Terminologie wurde präzisiert“21. Einige Abschnitte von Kants Schriften wurden de facto aufs Neue übersetzt, andere korrigiert, teilweise wurden Kommentare neu geschrieben.

Wenn die Kant-Ausgabe der 60er Jahre die ,Verdrehungen' der kantischen Terminologie durch die Anhänger der idealistischen Philosophie tadelte, sind eben diese ,Verdrehungen' in der Ausgabe Gulygas zur, Klassik' stilisiert. Die kommentatorischen und editorischen Errungenschaften der sowjetischen Zeit wurden von Gulyga bei dieser Wiederherstellung zum Teil ignoriert. Dennoch ist die Jubiläumsausgabe 1994 bis heute die vollständigste russische und wohl die verbreitetste. Aber es fehlen in ihr ebenso wie in der Ausgabe der 60er Jahre einige Arbeiten Kants (z. B. De igne), andere sind nur lückenhaft veröffentlicht (Die wahre Schätzung der lebendigen Kräfte, Der Streit der Fakultäten). Da die meisten Übersetzungen eine lange Vorgeschichte hatten und aus verschiedenen Quellen stammten, führte das trotz aller Versuche, die Terminologie zu präzisieren, zu einem gewissen Durcheinander von verschiedenen Bänden und verschiedenen Schriften Kants.

Anfang der 90er Jahre des 20. Jahrhunderts wurde eine neue zweisprachige Kant-Ausgabe, herausgegeben von Nelly Wasilewna Motroschilowa

${ }^{18}$ Immanuil Kant, Sochineniya v vosmi tomakh, ed. Arseniy Vladimirovich Gulyga (Moskau: ChORO, 1994).

${ }^{19}$ Vor allem in der Zeitschrift Voprosy filosofii und im Jahrbuch Kantovskiy sbornik.

${ }^{20}$ Vgl. Arseniy Vladimirovich Gulyga, "Revolyutsiya dukha (Zhizn i tvorchestvo Immanuila Kanta)", in: Immanuil Kant, Sochineniya v vosmi tomakh, Bd. 1, 5-49.

${ }^{21}$ Kant, Sochineniya $v$ vosmi tomakh, Bd. 1, 4. 
und Burkhard Tuschling (1937-2012), begonnen ${ }^{22}$. Im ersten Band der bilateralen Ausgabe unterstrich Motroschilowa die Einmaligkeit einer solchen Ausgabe in der damaligen Kantforschung ${ }^{23}$. Sie argumentierte zugunsten der These der „unvergänglichen und sogar zunehmenden Aktualität von Kants Philosophie ${ }^{\text {"24 }}$ und kam zu dem Schluss: Die Texte des scharfsinnigen Kant „in die Händen des denkenden Lesers, vor allem des russischen Lesers, zu geben, heißt, ihn bei der für unsere Zeit wesentlichen Suche geistiger Art

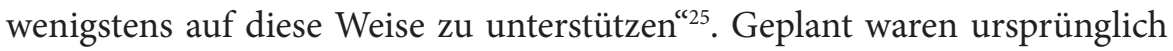
nur vier Bände, aber 2019 ist der fünfte und der letzte Band dieser Ausgabe erschienen. Im Unterschied zu den vorherigen russischen Ausgaben enthält die zweisprachige Ausgabe nicht nur einen sehr ausführlichen wissenschaftlichen Apparat einschließlich Lesarten, sondern auch zahlreiche Kommentare sowohl zu der neuen Redaktion der Übersetzung als auch zu der russischen Rezeption der publizierten Texte. Manchmal sind diese Materialien umfangreicher als der Text von Kant selbst. Vor allem die neuen Übersetzungen der Grundlegung zur Metaphysik der Sitten, der Kritik der praktischen Vernunft (herausgegeben von Andrej Konstantinovič Sudakov und Erich Jur'evič Solov'ev) und der Kritik der Urteilskraft (herausgegeben von Motroschilowa und Tamara Borisovna Dlugač) nahmen allen vorherigen russischen Übersetzungen dieser Werke de facto ihre Aktualität. Bei anderen Übersetzungen dieser Edition ist der Kontrast zu den früheren Publikationen vielleicht nicht so groß. Aber die zweisprachige Ausgabe enthält nur eine Auswahl der Schriften Kants. Sowohl die alte Kant-Ausgabe der 60er Jahre wie auch die Jubiläumsausgabe ist vollständiger. Darüber hinaus zeichnen leider auch für die zweisprachige Kant-Ausgabe verschiedene Inkonsequenzen aus. Das bezieht sich z.B. auf die Metaphysischen Anfangsgründe der Rechtslehre und die Metaphysischen Anfangsgründe der Tugendlehre im 5. Band.

${ }^{22}$ Immanuil Kant, Sochineniya na nemetskom i russkom yazykakh, ed. Nelli Vasilyevna Motroshilova, Burkhard Tuschling, Bd. 1-5 (Moskau: Kami, Nauka, Moskovskiy filosofskiy fond, Kanon, 1994-2019).

${ }^{23}$ Vgl. Nelli Vasilyevna Motroshilova, "Predislovie”, in: Immanuil Kant, Sochineniya na nemetskom i russkom yazykakh, ed. Motroshilova, Tuschling, Bd. 1 (Moskau: Kami, 1994), 42.

${ }^{24}$ Motroshilova, "Predislovie", 58.

${ }^{25}$ Ibidem, 59. 
In den letzten Jahren sind noch einige neue Übersetzungen Kants erschienen, die in keiner dieser Ausgaben zu finden sind: Ausschnitte aus dem handschriftlichen Nachlass Kants ${ }^{26}$, die Philosophische Religionslehre von Pölitz ${ }^{27}$, Vorlesungen über die Ethik ${ }^{28}$, Ausschnitte aus den Vorlesungen über das Naturrecht ${ }^{29}$ und einige Briefe ${ }^{30}$.

Die neue zweisprachige Kant-Ausgabe schuf aber eine gute Grundlage für eine weitere Editionsarbeit. Die russischen Schriften Kants benötigen eine gewisse Vereinheitlichung und Präzisierung der Terminologie, die lückenhaften Publikationen eine vollständige Übersetzung und ein Teil der noch nicht übersetzen Texte eine neue Übersetzung. Obwohl der letzte Band der Kant-Ausgabe erst 2019 erschienen ist, braucht die russische Kantforschung im Prinzip bereits jetzt eine neue Kant-Ausgabe.

\section{Einige Schwierigkeiten der Übersetzung Kants ins Russische und ihre Ursachen}

Die zahlreichen Schwierigkeiten, die bei einer Übersetzung Kants ins Russische entstehen, sind unterschiedlicher Natur. Einige davon sind wohl auch für die Übersetzungen in andere europäische Sprachen charakteristisch, aber es gibt eine ganze Reihe von Problemen, die gerade für die russische Sprache kennzeichnend sind. So ist es z.B. unmöglich, Kants Begriffe im Plural und im Singular einfach auf Russisch wiederzugeben. Das russische Standardwort für ,Pflicht', ,dolg', etwa bedeutet nämlich im Plural ausschließlich Schulden.

\footnotetext{
${ }^{26}$ Immanuil Kant, Iz rukopisnogo naslediya (materialy $k$ „Kritike chistogo razuma“, Opus postumum), ed. Vladimir Aleksandrovich Zhuchkov (Moskau: Nauka, 2000).

${ }^{27}$ Immanuil Kant, Lektsii po etike, ed. Abdusalam Abdulkerimovich Guseynov (Moskau: Respublika, 2000).

${ }^{28}$ Immanuil Kant, Lektsii o filosofskom uchenii o religii (redaktsiya K. G. L. Pelitsa), transl. Lyudmila Eduardovna Kryshtop, ed. Aleksey Nikolayevich Kruglov (Moskau: Kanon, 2016).

${ }^{29}$ Immanuil Kant, "Estestvennoye pravo Feyerabenda", transl. Lyudmila Eduardovna Kryshtop, Kantovskiy sbornik 35, 3 (2016): 75-81; 35, 4 (2016): 55-62.

${ }^{30}$ Vor allem, der Briefwechsel Kants mit Johann Georg Hamann: "Perepiska Immanuila Kanta i Ioganna Georga Gamana”, transl. Vladimir Khamitovich Gilmanov, Kantovskiy sbornik 29, 1 (2010): 90-111.
} 
Außerdem besitzt die russische Sprache manchmal keine Entsprechungen für Kants Gegenüberstellungen. So hat bis heute kein Übersetzer den Unterschied zwischen ,Gemüt' und ,Seele' adäquat zu übertragen verstanden, denn es gibt dafür im Russischen nur ein einziges Wort - ,duša. Ein ähnliches Problem existiert im Russischen für die kantische Unterscheidung von ,Gerechtigkeit' und ,Billigkeit', obwohl hier bereits Lösungen vorgeschlagen wurden $^{31}$. Des Weiteren gibt es im Russischen manchmal verschiedene Übersetzungsmöglichkeiten, und die Entscheidung für die eine oder andere Variante ist bereits eine Interpretation. $\mathrm{Zu}$ einem solchen Streitpunkt ist in der neuen zweisprachigen Kant-Ausgabe der Begriff ,Handlung' geworden, der in der Grundlegung zur Metaphysik der Sitten ausschließlich als ,dejstvie' übersetzt wurde, während in der Kritik der praktischen Vernunft und in der Metaphysik der Sitten dafür auch ,postupok' gebraucht wurde. Ein anderes Beispiel solcher Art ist die Übersetzung des Wortes ,Knecht' als ,rab', sluga' oder, cholop'. In einem vierten Fall stimmt zwar die Zahl der Entsprechungen mit dem kantischen Original überein, aber die russischen Begriffe bilden inhaltlich eine andere Reihe: Bis jetzt ist es keinem gelungen, die kantische Begriffstroika Glück - Glückseligkeit - Seligkeit adäquat ins Russische zu übersetzen. Zwar kann man für jeden einzelnen Begriff verschiedene Varianten finden. Es ergibt sich aber für die ganze Reihe nie der gleiche Sinn wie im Deutschen, weil der Zusammenhang der Worte im Russischen anders gedacht wird. Ein seltener russischer Vorname lautet Fortunat, zwei andere sind verbreiteter, nämlich Felix und Makar. Das kann man aber nicht auf die Begriffe übertragen. So kann man leider nicht einfach ,udača/vezenie' für ,Glück', sčast'e' für ,Glückseligkeit' und ,blaženstvo' für ,Seligkeit' verwenden. Fast ein halbes Jahrhundert lang wurde in der russischen Kantforschung heftig darüber diskutiert, wie das kantische, Ding an sich' ins Russische zu übersetzen sei, und die zweisprachige Kant-Ausgabe beschwor diese Auseinandersetzungen wieder herauf ${ }^{32}$.

${ }^{31}$ Vgl. dazu die Übersetzungen und die Aufsätze von L. E. Krystop.

${ }^{32}$ Vgl. Nelli Vasilyevna Motroshilova, “Kommentarii k novoy redaktsii perevoda „Kritiki chistogo razuma “", in: Immanuil Kant, Sochineniya na nemetskom i russkom yazykakh, ed. Motroshilova, Tuschling, Bd. 2.2 (Moskau: Nauka, 2006), 699-715; Viktor Igorevich Molchanov, "Bezotnositelnoye i nezavisimoye. O razlichii terminov i slov v „Kritike chistogo razuma“ Kanta”, Istoriko-filosofskiy ezhegodnik 32 (2017): 103-133; Aleksey Nikolayevich Kruglov, 
Einige Probleme der Übersetzung Kants ins Russische und ihre Ursachen möchte ich in drei Beispielen ausführlicher erläutern.

\section{a) Die Kausalität der Ursache}

Das erste Beispiel ist Kants Ausdruck ,Kausalität der Ursache', der sowohl in der Kritik der reinen Vernunft als auch in den Prolegomena vorkommt. Die Schwierigkeiten in der Übersetzung wurden zum wesentlichen Teil dadurch verursacht, dass die Übersetzer den Sinn des kantischen Ausdrucks nicht so richtig verstanden. Erst neuere Studien zur Vorgeschichte des Kausalitätsbegriffs, der zu Kants Zeiten noch relativ neu und eben durch Kant erst populär gemacht worden war, verdeutlichten die Rolle von Christian August Crusius und den kosmologischen Kontext von Kants Gedanken, die lange in einer eher erkenntnistheoretischen Perspektive aufgefasst worden waren ${ }^{33}$.

Im Beweis der Thesis der dritten Antinomie der Kritik der reinen Vernunft wurde der Ausdruck, Kausalität der Ursache' ${ }^{34}$ folgendermaßen übersetzt:

,pričina' (Vladislavlev 1867) ${ }^{35}$,

,pričinnost' pričiny' (Sokolov 1896-1897, 1902; Losskij 1907, 1915) ${ }^{36}$,

,kauzal'nost' pričiny' (Kant-Ausgabe 1964, Kant-Ausgabe 1994, Kritik der reinen Vernunft 1994, Kant-Ausgabe 2006) ${ }^{37}$.

Die Variante von Michail Ivanovič Vladislavlev (1840-1890), dem ersten Übersetzer der Kritik der reinen Vernunft ins Russische, zeigt, dass er

"O nekotorykh istochnikakh i problemakh perevoda kantovskogo termina „Ding an sich“", Istoriko-filosofskiy ezhegodnik 33 (2017): 137-164.

${ }^{33}$ Vgl. dazu ausführlicher Alexei N. Krouglov, “Zum Begriff der Kausalität bei I. Kant”, in: Kant im Spiegel der russischen Kantforschung heute, ed. Norbert Hinske, Nelly Motroschilowa (Stuttgart-Bad Cannstatt: frommann-holzboog, 2008), 53-69.

${ }^{34}$ Vgl. Immanuil Kant, Kritik der reinen Vernunft, B 472/A 444.

${ }^{35}$ Idem, Kritika chistago razuma, transl. Vladislavlev, 368.

${ }^{36}$ Idem, Kritika chistago razuma, transl. Sokolov (Sankt-Petersburg: Popov, 1902), 318; Kant, Kritika chistago razuma, transl. Losskiy (Sankt-Petersburg: Tipografiya Stasyulevicha, 1907), 274; Kant, Kritika chistago razuma, transl. Losskiy (Petrograd: Tipografiya Stasyulevicha, 1915), 378, 380.

${ }^{37}$ Kant, "Kritika chistogo razuma", transl. Losskiy, Arzakanyan, Itkin, 418; Kant, Kritika chistogo razuma, new transl. Losskiy, Arzakanyan, Itkin, 280; Kant, "Kritika chistogo razuma", transl. Losskiy, Arzakanyan, Itkin, Adreeva, 350; Kant, "Kritika chistogo razuma", transl. Losskiy, Arzakanyan, Itkin, Motroshilova, Bd. 2.1, 597. 
den Ausdruck ,Kausalität der Ursache' einfach mit ,Ursache' identifizierte. Nur Losskij war konsequent und übersetzte ,Kausalität' sowohl als einzelnen Begriff als auch in der Wortverbindung, Kausalität der Ursache' immer als ,pričinnost", d. h. als Ursächlichkeit. In den meisten Ausgaben entstand aber eine gewisse Gegenüberstellung zwischen einer Ursächlichkeit und einer Kausalität: Die ,Kausalität' in der Kategorientafe ${ }^{38}$ oder in der zweiten Analogie der Erfahrung der zweiten Auflage ${ }^{39}$ wurde als ,pričinnost" ${ }^{\prime 0}$ und im Ausdruck ,Kausalität der Ursache' plötzlich als ,kauzal'nost” übersetzt. Die zweisprachige Kant-Ausgabe korrigierte diese Inkonsequenz: Hier wird ,Kausalität' konsequent als ,kauzal'nost” wiedergeben ${ }^{41}$.

In den Prolegomena ist in Zusammenhang mit der Kausalität der 53. Paragraph von Interesse. Kants Ausdruck „Bestimmung der Causaltät ihrer Ursache “42 wird hier so übersetzt:

„kauzal'noe opredelenie ego pričiny“43,

„opredelenie kauzal'nosti ego pričiny“" ${ }^{44}$.

${ }^{38}$ Vgl. Kant, Kritik der reinen Vernunft, B 106/A 80.

${ }^{39}$ Vgl. ibidem, B 232.

${ }^{40}$ Kant, Kritika chistago razuma, transl. Vladislavlev, 74, 175; Kant, Kritika chistago razuma, transl. Sokolov (Sankt-Petersburg: Popov, 1902), 94, 171; Kant, Kritika chistago razuma, transl. Losskiy (Sankt-Petersburg: Tipografiya Stasyulevicha, 1907), 83, 152; Kant, Kritika chistago razuma, transl. Losskiy (Petrograd: Tipografiya Stasyulevicha, 1915), 119, 210; Kant, "Kritika chistogo razuma", transl. Losskiy, Arzakanyan, Itkin, 175, 258; Kant, Kritika chistogo razuma, new transl. Losskiy, Arzakanyan, Itkin, 86, 152; Kant, "Kritika chistogo razuma", transl. Losskiy, Arzakanyan, Itkin, Adreeva, 110, 193.

${ }^{41}$ Kant, "Kritika chistogo razuma", transl. Losskiy, Arzakanyan, Itkin, Motroshilova, Bd. 2.1, 173, 321, 597.

${ }^{42}$ Immanuel Kant, "Prolegomena zu einer jeden künftigen Metaphysik, die als Wissenschaft wird auftreten können”, in: Kant's gesammelte Schriften, ed. Königlich Preußische Akademie der Wissenschaften, Bd. IV (Berlin: Georg Reimer, 1914), 343.

${ }^{43}$ Kant, Prolegomeny ko vsyakoy budushchey metafizike, mogushchey vozniknut v smysle nauki, transl. Vladimir Sergeyevich Solovyev (Sankt-Petersburg: Tipografiya Gattsuka, 1889), 136.

${ }^{44}$ Kant, Prolegomeny, transl. Solovyev, Fokht, ed. Saradzhev, 239; Kant, Prolegomeny ko vsyakoy budushchey metafizike, mogushchey vozniknut $v$ kachestve nauki, transl. Solovyev, Fokht, Dynnik, 125; Kant, "Prolegomeny ko vsyakoy budushchey metafizike, mogushchey poyavitsya kak nauka", transl. Solovyev, Fokht, Dynnik, Itkin, in: Kant, Sochineniya $v$ shesti tomakh, ed. Asmus, Gulyga, Oyzerman, Bd. 4(1) (Moskau: Mysl, 1965), 165; Kant, "Prolegomeny ko vsyakoy budushchey metafizike, kotoraya mozhet poyavitsya kak nauka", transl. 
Kants Ausdruck „Bestimmung der Ursache zur Kausalität“45 wird im Russischen folgendermaßen wiedergeben:

„opredelenie pričiny k dejstvovaniju“"46,

„opredelenie pričiny k kauzal'nosti“" ${ }^{“ 4}$.

Die Wortverbindung „Causalität der Ursache“48 sieht in verschiedenen Übersetzungen so aus:

„proizvoditel'nost' pričiny“4 ${ }^{4}$,

„proizvodjaščaja sila pričiny“"50,

„proizvoditel'nost' (kauzal'nost') pričiny “51,

„kauzal'nost' pričiny“" ${ }^{2}$.

Kants Ausdruck „die Ursache ihrer Causalität nach“53 wird aber anders übersetzt:

Solovyev, Fokht, Dynnik, Itkin, Belyaev, in: Kant, Sochineniya v vosmi tomakh, ed. Gulyga, Bd. 4 (Moskau: ChORO, 1994), 106.

${ }^{45}$ Kant, "Prolegomena zu einer jeden künftigen Metaphysik, die als Wissenschaft wird auftreten können”, 343.

${ }^{46}$ Idem, Prolegomeny ko vsyakoy budushchey metafizike, mogushchey vozniknut $v$ smysle nauki, transl. Solovyev, 136; Kant, Prolegomeny, transl. Solovyev, Fokht, ed. Saradzhev, 239; Kant, Prolegomeny ko vsyakoy budushchey metafizike, mogushchey vozniknut v kachestve nauki, transl. Solovyev, Fokht, Dynnik, 125; Kant, "Prolegomeny ko vsyakoy budushchey metafizike, mogushchey poyavitsya kak nauka", transl. Solovyev, Fokht, Dynnik, Itkin, 166.

${ }^{47}$ Kant, "Prolegomeny ko vsyakoy budushchey metafizike. kotoraya mozhet poyavitsya kak nauka", transl. Solovyev, Fokht, Dynnik, Itkin, Belyaev, 106.

${ }^{48}$ Kant, "Prolegomena zu einer jeden künftigen Metaphysik, die als Wissenschaft wird auftreten können", 344.

${ }^{49}$ Idem, Prolegomeny ko vsyakoy budushchey metafizike, mogushchey vozniknut v smysle nauki, transl. Solovyev, 136.

${ }^{50}$ Kant, Prolegomeny ko vsyakoy budushchey metafizike, mogushchey vozniknut $v$ smysle nauki, transl. Solovyev, 136; Kant, Prolegomeny, transl. Solovyev, Fokht, ed. Saradzhev, 239; Kant, Prolegomeny ko vsyakoy budushchey metafizike, mogushchey vozniknut v kachestve nauki, transl. Solovyev, Fokht, Dynnik, 126.

${ }^{51}$ Kant, Prolegomeny, transl. Solovyev, Fokht, ed. Saradzhev, 239; Kant, Prolegomeny ko vsyakoy budushchey metafizike, mogushchey vozniknut v kachestve nauki, transl. Solovyev, Fokht, Dynnik, 125.

${ }^{52}$ Kant, "Prolegomeny ko vsyakoy budushchey metafizike. mogushchey poyavitsya kak nauka", transl. Solovyev, Fokht, Dynnik, Itkin, 166; Kant, "Prolegomeny ko vsyakoy budushchey metafizike. kotoraya mozhet poyavitsya kak nauka", transl. Solovyev, Fokht, Dynnik, Itkin, Belyaev, 106.

${ }^{53}$ Kant, "Prolegomena zu einer jeden künftigen Metaphysik, die als Wissenschaft wird auftreten können”, 344. 
„pričina v svoej dejstvujuščej sile“"54,

"pričina po svoej kauzal'nosti“" ${ }^{\text {“55. }}$.

Abgesehen von der Originalübersetzung oder, sozusagen, interpretierenden Übersetzung von Solov'ev war und wird Kants Terminus ,Kausalität' in den Prolegomena also am häufigsten mit „kauzal'nost““ und nicht mit „pričinnost" "übersetzt, wie es lange in der russischen Kritik der reinen Vernunft der Fall war. Ob die Variante Solov'evs auch heute sowohl für die Prolegomena als auch für die Kritik der reinen Vernunft zu halten ist, darüber lässt sich streiten. Jedenfalls sind die Korrekturen der Übersetzung Solov'evs, die nach seinem Tod zuerst in der Sowjetunion und dann auch im postsowjetischen Russland vorgenommen wurden, ziemlich fragwürdig. Erstens sind sie vom Standpunkt der philologischen Strenge aus nicht konsequent durchgeführt worden, denn der Terminus, Kausalität' wird ab und zu nach wie vor als „pričinnost“" übersetzt. Zweitens klingt der Ausdruck „kauzal'nost' pričiny“ zwar besser als "pričinnost' pričiny“, doch gibt diese Wortverbindung den Sinn der kantischen Philosophie kaum mit solcher Verständlichkeit und Überzeugungskraft wieder wie diejenige Variante, die Solov'ev vorschlug.

Die Diskrepanz zwischen den russischen Ausgaben der Prolegomena und der Kritik der reinen Vernunft bei denselben Begriffen kann man relativ leicht erklären. Während alle gegenwärtigen Übersetzungen der Kritik der reinen Vernunft in der Tradition von Losskij stehen, lehnen sich alle russischen Ausgaben der Prolegomena an die Übersetzung von Solov'ev an. Und selbst in der Kant-Ausgabe der 60er Jahre und der Jubiläumsausgabe von 1994 wurden sie in verschiedenen Bänden und von verschiedenen Kantforschern ediert und herausgegeben. Ein weiteres interessantes Detail ist die Tatsache, dass nach den drei Auflagen der Übersetzung der Prolegomena von Solov'ev ( ${ }^{1} 1889$, $\left.{ }^{2} 1892,{ }^{3} 1905\right)$ in der sowjetischen und postsowjetischen Zeit vier andere

${ }^{54}$ Idem, Prolegomeny ko vsyakoy budushchey metafizike, mogushchey vozniknut $v$ smysle nauki, transl. Solovyev, 136-137; Kant, Prolegomeny, transl. Solovyev, Fokht, ed. Saradzhev, 239-240; Kant, Prolegomeny ko vsyakoy budushchey metafizike, mogushchey vozniknut v kachestve nauki, transl. Solovyev, Fokht, Dynnik, 126.

${ }^{55}$ Kant, "Prolegomeny ko vsyakoy budushchey metafizike. mogushchey poyavitsya kak nauka", transl. Solovyev, Fokht, Dynnik, Itkin, 166; Kant, "Prolegomeny ko vsyakoy budushchey metafizike, kotoraya mozhet poyavitsya kak nauka", transl. Solovyev, Fokht, Dynnik, Itkin, Belyaev, 106. 
Ausgaben der Prolegomena erschienen sind. Und jede neue Ausgabe nach Solov'ev hat einen anderen Titel:

Prolegomeny ko vsjakoj buduščej metafizike, moguščej vozniknut'v smysle nauki $(1889,1892,1905)$;

Prolegomeny (1934);

Prolegomeny ko vsjakoj buduščej metafizike, moguščej vozniknut'v kačestve nauki (1937);

Prolegomeny ko vsjakoj buduščej metafizike, moguščej pojavit’sja kak nauka (1965);

Prolegomeny ko vsjakoj buduščej metafizike, kotoraja možet pojavitsja kak nauka (1994).

\section{b) Gesinnung}

In der Ethik und in der Religionsphilosophie Kants hat in der kritischen Zeit der Begriff ,Gesinnung' eine enorme Bedeutung. Seine Übersetzung in andere europäische Sprachen bereitet allerdings zahlreiche Probleme ${ }^{56}$. So tauchen in den englischsprachigen Übersetzungen ,disposition', attitude, ,conviction', , sentiment', ,comportment of mind', ,intention' und ,Gesinnung' als Entsprechungen für das deutsche Wort Gesinnung auf. Noch bunter sind die Varianten der russischen Übersetzungen. In den vorkritischen Schriften Kants ist das Wort ,Gesinnung' noch kein feststehender Terminus. Für die Übersetzung ins Russische werden Begriffe wie ,nastroenie', ,stremlenie,' ,naklonnost", ,ubeždenie,, ,dobrodetel”, ,dobrodetel'nost" und ,obraz myslej' verwendet.

Was die Grundlegung zur Metaphysik der Sitten, die Kritik der praktischen Vernunft und die Religion innerhalb der Grenzen der bloßen Vernunft betrifft, sieht die Situation ähnlich aus, obwohl einer der bedeutendsten

${ }^{56}$ Vgl. Leticia Machado Pinheiro, "O conceito kantiano de intenção,Gesinnung em ,Sobre o mal radical na natureza humana", Revista Portuguesa de Filosofia 61 (2005): 1019-1026; Krystop, Ludmila Eduardovna, "Etika chistogo obraza mysley Kanta”, in: Istoriya filosofii i sotsiokulturnyy kontekst. Materialy Mezhdunarodnoy konferentsii. Moskva. 24-25 dekabrya 2012 g., ed. Taras Aleksandrovich Shiyan, Moskau: RGGU, 2012, 158-167; Stephen R. Palmquist, "What is Kantian Gesinnung? On the Priority of Volition over Metaphysics and Psychology in Religion within the Bounds of Bare Reason", Kantian Review 20-22 (2015): 235-264. 
russischen Kantforscher der sowjetischen Zeit Valentin Ferdinandovič Asmus (1894-1975) sich in der Kant-Ausgabe der 60er Jahre bemüht hatte, die Übersetzungen zu vereinheitlichen. Er ging dabei bis zu einem gewissen Grade in die Richtung der späteren polnischen Übersetzung der Religion innerhalb der Grenzen der bloßen Vernunft von Aleksandr Bobko (,usposobienie'). Die Entscheidung von Asmus war, Gesinnung mit ,ubeždenie', zu Deutsch Überzeugung, zu übersetzen ${ }^{57}$. Die einheitlichen vorrevolutionären Varianten waren dagegen das heute veraltete Wort ,čuvstvovanie ${ }^{58}$ und ,nastroenie', zu Deutsch Stimmung ${ }^{59}$. Die Kritik der praktischen Vernunft in der zweisprachigen Ausgabe (herausgegeben von Erich Jur'evič Solov'ev) und die Religion innerhalb der Grenzen der bloßen Vernunft in der Jubiläumsausgabe (in der Redaktion von Aleksandr Viktorovič Michajlov [1938-1995]) plädieren für eine situationsabhängige kontextuelle Übersetzung des Begriffs ,Gesinnung, so dass nach dem russischen Text kaum zu erraten ist, ob im Original ,Gesinnung', ,Überzeugung', ,Denkungsart' oder ,Sinnesänderung' steht.

Insgesamt finden sich folgende russische Varianten für den Ausdruck ,Gesinnung' bei Kant: ,stremlenie', ,naklonnost”, ,namerenie', ,čuvstvovanie', ,dobrodetel”, ,dobrodetel'nost”, , ubeždenie', ,obraz myslej', ,mysli', ,nastroenie', ,nastroennost" und ,umonastroenie. Besonders schwierig ist es in russischen Übersetzungen, zwischen Gesinnung und Denkungsart zu unterscheiden. Dabei war es Kants selbst, der zu dieser Ungenauigkeit in der Religion innerhalb der Grenzen der bloßen Vernunft beitrug. Aber neben der Synonymität dieser Begriffe finden sich bei Kant auch gewisse Unterschiede. Erstens enthält der Begriff, ,Denkungsart' keinen so evidenten Willensaspekt wie es bei ,Gesinnung' der Fall ist. Während sich zweitens im Begriff der ,Gesinnung' in der Grundlegung zur Metaphysik der Sitten und in der Kritik der praktischen Vernunft Sinnliches und Intellektuelles vereinigen, bezieht sich der Begriff der ,Denkungsart' doch ausschließlich auf das Intellektuelle. Drittens gehört der Begriff der ,Gesinnung' in der Religion innerhalb der Grenzen der bloßen Vernunft dem Bereich des Übersinnlichen an. Viertens bedeutet der

${ }^{57}$ Immanuil Kant, "Osnovy metafiziki nravstvennosti”, in: Immanuil Kant, Sochineniya $v$ shesti tomakh, Bd. 4(1), ed. Asmus, 243, 250, 255, 277, 278.

${ }^{58}$ Kantovo osnovaniye dlya metafiziki nravov, 36, 51, 61, 109, 110.

${ }^{59}$ Vgl. Immanuil Kant, Osnovopolozheniye k metafizike nravov, transl. L. D. B., ed. Veniamin Mikhaylovich Khvostov (Moskau: Tipografiya Vilde, 1912), 26, 33, 39, 64. 
Begriff, Denkungsart' bei Kant ab und zu so etwas wie Methode der Interpretation oder der Deutung, was für, Gesinnung' gar nicht zutrifft.

Aus diesen Gründen, aber auch aufgrund der Vorgeschichte des Begriffs, in der Sinnliches und Intellektuelles dem Kern der Bedeutung angehörten, scheint es mir angemessen, in den kritischen Schriften Kants den Begriff, Gesinnung' konsequent als, umonastroenie' zu übersetzen. Es wurde diese Variante bereits gelegentlich in der Grundlegung zur Metaphysik der Sitten, der Kritik der praktischen Vernunft und der Religion innerhalb der Grenzen der bloßen Vernunft verwendet ${ }^{60}$. Dieselbe Variante wird auch einheitlich in der jüngsten Übersetzung des Naturrechts Feyerabend gebraucht ${ }^{61}$. Und noch früher wurde, wenn auch nicht immer und überall, der Begriff ,Gesinnung' bei Hegel als , umonastroenie' ins Russische übersetzt ${ }^{62}$. Bei einer neuen Übersetzung der Religion innerhalb der Grenzen der bloßen Vernunft, die aktuell als Herausforderung vor der russischen Kantforschung steht, muss das Problem der Übersetzung des Ausdrucks, Gesinnung' ins Russische gelöst werden, das früher überhaupt nicht als solches wahrgenommen wurde.

\section{c) Der Brief Kants an die Zarin Elisabeth}

Im Dezember 2018 ist eine kurze Rede des Vize-Admirals Igor Timerbulatovič Muchametšin, damals Stabschef der Baltischen Flotte, vor den Marinenoffizieren zu einem viel diskutierten Vorfall geworden, die er über „einen gewissen, nicht unbekannten Immanuel Kant“ hielt: „... alle sagen: Kant, Kant, und noch irgendetwas. Dieser Mensch hat seine Heimat

${ }^{60}$ Vgl. Immanuel Kant, “Osnovopolozheniye k metafizike nravov”, transl. Andrey Konstantinovich Sudakov, in: Immanuel Kant, Sochineniya na nemetskom i russkom yazykakh, ed. Nelli Vasilyevna Motroshilova, Burkhard Tuschling, Bd. 3 (Moskau: Moskovskiy filosofskiy fond, 1997), 189; Immanuel Kant, "Religiya v predelakh tolko razuma", in: Immanuel Kant, Sochineniya v vosmi tomakh, ed. Arseniy Vladimirovich Gulyga, Bd. 6 (Moskau: ChORO, 1994), 191, 219.

${ }^{61}$ Vgl. Immanuel Kant, "Estestvennoye pravo Feyerabenda", Kantovskiy sbornik 35, 4 (2016): 60-62.

${ }^{62}$ Vgl. z.B. Georg Vilgelm Fridrikh Gegel, Filosofiya prava, transl. Boris Grigoryevich Stolpner, Margarita Iosifovna Levina (Moskau: Mysl, 1990), 292, § 268; Georg Vilgelm Fridrikh Gegel, "Filosofskaya propedevtika", in: Georg Vilgelm Fridrikh Gegel, Raboty raznykh let, Bd. 2 (Moskau: Mysl, 1971), 55, § 32-33; 34, § 34. 
verraten, sich erniedrigt, ist auf den Knien gekrochen, um einen Lehrstuhl an der Universität zu bekommen - damit er dort unterrichten, irgendwelche unverständlichen Bücher schreiben kann, die keiner von denen, die hier stehen, je gelesen hat und auch nie lesen wird... ${ }^{\text {“63. }}$. Es kam nicht von ungefähr zu dieser Rede, denn sie reproduziert einige typische Vorurteile über Kant, die in Russland schon im 19. Jahrhundert kursierten, obwohl sie damals eher marginal waren. Interessant ist die Rede aber unter anderem, weil sie ein akutes Problem der russischen Kantforschung evident macht. Der Vize-Admiral rekurriert hier auf eine schlechte Übersetzung von Kants Brief an die russische Zarin Elisaveta Petrovna (1709-1762), die der Königsberger PrivatDozent 1758 im russischen Königsberg zur Zeit des Siebenjährigen Krieges schrieb. Nach dem Tod des Königsberger Professors Johann David Kypke wollte Kant dessen Lehrstuhl der Logik und Metaphysik übernehmen ${ }^{64}$.

Sein Brief an die russische Zarin vom 14. Dezember 1758 beendete Kant mit folgenden Worten: „Ich ersterbe in tiefester devotion Ew. Kayserl Majestät allerunterthänigster Knecht Immanuel Kant" ${ }^{\text {"65. }}$. Zwei Jahre vorher, als Königsberg noch preußisch war, versuchte Kant ebenfalls eine außerplanmäßige Professur an der Universität zu erhalten, weshalb er an den preußischen König Friedrich den Großen einen Brief schrieb, an dessen Ende ebenfalls steht: „...und ersterbe in tiefster Unterthänigkeit Ew: Königl: Majestät allerunterthänigster Knecht Immanuel Kant "66. Auch der Brief an Friedrich den Großen aus dem Jahre 1770, nach dem Kant endlich die Professur an der Universität Königsberg erhalten hatte, endet wie folgt: „Ich ersterbe in tiefster devotion

${ }^{63} \mathrm{Vgl}$. https://www.newkaliningrad.ru/news/briefs/community/21287161-pisal-kakie-toneponyatnye-knigi-vitse-admiral-baltflota-rasskazal-moryakam-o-kante-video.html, 20.03.2020.

${ }^{64}$ Vgl. dazu ausführlicher Aleksey Nikolayevich Kruglov, ",Vashego Imper. Velichestva vsepoddanneyshiy rab Immanuil Kant': o nekotorykh trudnostyakh perevoda pisma Kanta k tsaritse Elizavete Petrovne", in: Transfer - Kultur - Akteur. Sbornik statey k 60-letiyu Dirka Kempera, ed. Natalia Aleksandrovna Bakshi, Aleksey Iosifovich Zherebin, Ivonne Pertsgen, Elizabet Shore (Moskau: RGGU, 2019), 197-203.

${ }^{65}$ Immanuel Kant, "Brief an die russische Kaiserin Elisabeth vom 14. Dezember 1758", in: Kant's gesammelte Schriften, ed. Königlich Preußische Akademie der Wissenschaften, Bd. X (Berlin: Georg Reimer, 1922), 6.

${ }^{66}$ Immanuel Kant, "Brief an Friedrich II. vom 8. April 1756”, in: Kant's gesammelte Schriften, ed. Königlich Preußische Akademie der Wissenschaften, Bd. X (Berlin: Georg Reimer, 1922), 3. 
als Ew. Königl: Majestät allerunterthänigster Knecht Immanuel Kant ${ }^{\text {“67 }}$. Zum ersten Mal aber taucht diese bürokratische Formel bei Kant schon in der Widmung an denselben König in seiner Schrift Allgemeine Naturgeschichte und Theorie des Himmels (1755) auf: „Ich ersterbe in tiefster Devotion, Ew. Königl. Majestät allerunterthänigster Knecht, [...] der Verfasser “68.

Die Briefe Kants an Friedrich den Großen sind nicht ins Russische übersetzt worden. Die Widmung aus der Schrift von 1755 ist nur in der Jubiläumsausgabe 1994 wiedergegeben. Der Brief Kants an die Zarin aber ist seit $1893^{69}$ einige Mal mit kleinen, aber wesentlichen Unterschieden ins Russische übersetzt worden, die verbreitetste und bekannteste Version enthält aber dieselbe Jubiläumsausgabe von Gulyga. Michail Michajlovič Filippov (1858-1903) schlug in seiner Biographie Kants die Übersetzung vor: „Ja uverjaju $v$ glubočajšem devotion Vašego Imperatorskago Veličestva vsepoddannejšij rab Emmanuil Kant ${ }^{\text {“70 }}$. Drei Jahre später veröffentlichte Jurij Petrovič Bartenev (1866-1908) den Brief in der Zeitschrift Russkij archiv in einer anderen Übersetzung: „Gotov umeret’ v moej glubočajšej predannosti vašego imperatorskago veličestva naivernopoddannejšij rab Emmanuel' Kant ${ }^{\text {“71. }}$. Berühmt ist dieser Brief jedoch in der Redaktion von Gulyga geworden: „Umolkaju v glubočajšem uničiženii, Vašego Imper. Veličestva vernopoddannejšij rab

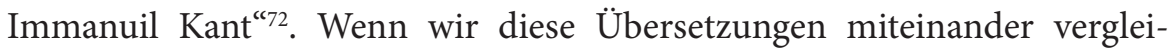
chen, sehen wir, dass vier Wörter aus dem Brief Kants als problematisch

${ }^{67}$ Immanuel Kant, "Brief an Friedrich II. vom 19. März 1770", in: Kant's gesammelte Schriften, ed. Königlich Preußische Akademie der Wissenschaften, Bd. X (Berlin: Georg Reimer, 1922), 93.

${ }^{68}$ Immanuel Kant, "Allgemeine Naturgeschichte und Theorie des Himmels", in: Kant's gesammelte Schriften, ed. Königlich Preußische Akademie der Wissenschaften, Bd. I (Berlin: Georg Reimer, 1910), 219.

${ }^{69}$ Ursprünglich in: Moskovskiye vedomosti, 10. April, no. 97 (1893): 5, später auch in: Syn Otechestva, 12. (24.) April, 98 (1893): 2.

${ }^{70}$ Vgl. Mikhail Mikhaylovich Filippov, Em. Kant. Ego zhizn i filosofskaya deyatelnost. Biograficheskiy ocherk (Sankt-Petersburg: Tipografiya Soykina, 1893), 22.

${ }^{71}$ Vgl. Yuriy Petrovich Bartenev, "Vsepoddanneysheye prosheniye filosofa Kanta imperatritse Elisavete Petrovne (1758)", Russkiy arkhiv, 7 (1896): 455-456.

${ }^{72}$ Immanuil Kant, "Pismo k imperatritse Elizavete Petrovne ot 14 dekabrya 1758 g." in: Immanuil Kant, Traktaty i pisma, ed. Arseniy Vladimirovich Gulyga (Moskau: Nauka, 1980), 505. Später veröffentlicht ohne Änderungen auch in: Immanuil Kant, Sochineniya v vosmi tomakh, ed. Arseniy Vladimirovich Gulyga, Bd. 8 (Moskau: ChORO, 1994), 464-465. 
erscheinen: ,ersterbe' als ,uverjaju', ,gotov umeret” und ,umolkaju'; ,devotion' als ,devotion', ,predannost" und ,uničiženie'; , allerunterthänigster' als ,vsepoddannejšij, ,naivernopoddannejšij' und ,vernopoddannejšij'; und ,Knecht' als ,rab' in allen drei Varianten.

Allein die Tatsache, dass Kant sich in dem Brief an die Zarin Elizaveta auf dieselbe Weise ausdrückte wie in dem an Friedrich den Großen, zeigt, dass es sich offensichtlich um eine Standardformel der bürokratischen Sprache des 18. Jahrhunderts handelt. Mehr noch, die Anleitung zum geistlichen Geschäfts-Style und zur geistlichen Geschäfts-Verwaltung aus dem Jahre 1828 empfahl den Ausdruck „Ich ersterbe in tiefster Devotion “73 auch im 19. Jahrhundert. Das Ende von Kants Brief erscheint als Klischee. Zu diesem Urteil kommt, wer den Briefwechsel jener Zeit durchblättert ${ }^{74}$.

Da Kant als Entsprechung für das lateinische Wort ,devotion' im Deutschen ,Unterthänigkeit' gebraucht, kann man mit guten Gründen die russische Variante ,predannost" dafür wählen. Obwohl die Bereitschaft zu sterben für das Verb ,ersterben' buchstäblich passt, ist in einem bürokratischen Brief die Übersetzung, smolkat" oder, umolkat" angemessener. Der Ausdruck, allerunterthänigster Knecht' ist auf Russisch im Briefwechsel Grigorij Aleksandrovič Potemkins (1739-1791) mit der Katharina der Großen (1729-1796) mit verschiedenen Abweichungen leicht zu finden: ,vsepoddannejšij, ,verno vsepoddannejšij, ,vernovsepoddannejšij, ,naivernejšij i vsepoddannejšij $\mathrm{rab}^{35}$. Aus diesen Gründen scheint mir folgende Übersetzung von Kants Brief

${ }^{73}$ Andreas Müller, Anleitung zum geistlichen Geschäfts-Style und zur geistlichen Geschäfts-Verwaltung sowohl nach der Pastoral und dem gemeinen Kirchenrechte als nach besonderen bayererischen Verordnungen. Nebst einem Anhange von Formularen aller Arten von Geschäfts-Aufsätzen, welche in d. verschiedenen Verzweigungen der geistlichen Amts-Verwaltung vorkommen, zunächst für katholische Geistliche (Würzburg: Etlinger, 1828), 52.

${ }^{74}$ Vgl. Günter Wagner, Die Sinfonien Carl Philipp Emanuel Bachs: werdende Gattung und Originalgenie (Stuttgart: Metzler, 1994), 14; Carl Mennicke, Hasse und die Brüder Graun als Symphoniker. Nebst Biographien und thematischen Katalogen (Leipzig: Breitkopf \& Härtel, 1906), 480; Zur Erinnerung an Gotthold Ephraim Lessing. Briefe und Aktenstücke aus den Papieren der Herzoglichen Bibliothek und den Akten des Herzoglichen Landeshauptarchivs zu Wolfenbüttel, ed. Otto von Heinemann (Leipzig: Hirzel, 1870), 75.

${ }^{75}$ Ekaterina II $i$ G. A. Potemkin. Lichnaya perepiska 1769-1791, ed. Vyacheslav Sergeyevich Lopatin (Moskau: Nauka, 1997), 5, 6, 11, 41, 149, 184, 185, 196, 197, 199 etc. 
an die Zarin Elizaveta richtig: „Smolkaju v glubočajšej predannosti Vašego Imper. Veličestva vsepoddannejšij rab Immanuil Kant ${ }^{\text {"776 }}$.

Gulyga, der in der Jubiläumsausgabe ,Unterthänigkeit' als eine gewisse Erniedrigung und ,allerunterthänigster' als ,getreuer allerunterthänigster' übersetzte, trug zu einem bitteren Beigeschmack bei, der in der Rede des Vize-Admirals Muchametšin zu hören ist. Das ist umso merkwürdiger, als in derselben Jubiläumsausgabe in einem anderen Band die Widmung Kants an Friedrich den Großen mit einem abweichenden Wortlaut übersetzt ist: „Prebyvaju $v$ glubočajšem blagogovenii. Vašego korolevskogo veličestva vsepoddannejšij sluga Avtor "77. Hätte der Vize-Admiral diese russische Übersetzung gehört, wäre Kant für ihn vermutlich zwar nach wie vor ein böser Geist, aber doch mit anderen Lastern.

\section{Bibliographie}

Bartenev Yuriy Petrovich. 1896. "Vsepoddanneysheye prosheniye filosofa Kanta imperatritse Elisavete Petrovne (1758)”. Russkiy arkhiv 7: 455-456.

Bykova Marina Fedorovna. 2015. "Novoye slovo v mirovom kantovedenii. O dvuyazychnom izdanii sochineniy Immanuila Kanta na nemetskom i russkom yazykakh". Istoriko-filosofskiy ezhegodnik: 362-380.

Ekaterina II i G. A. Potemkin. Lichnaya perepiska 1769-1791. 1997. Ed. Vyacheslav Sergeyevich Lopatin. Moskau: Nauka.

Filippov Mikhail Mikhaylovich. 1893. Em. Kant. Ego zhizn i filosofskaya deyatelnost. Biograficheskiy ocherk. Sankt-Petersburg: Tipografiya Soykina.

Gegel Georg Vilgelm Fridrikh. 1971. "Filosofskaya propedevtika”. In: Gegel Georg Vilgelm Fridrikh. Raboty raznykh let. Bd. 2. Moskau: Mysl, 7-212.

Gegel Georg Vilgelm Fridrikh. 1990. Filosofiya prava. Transl. Boris Grigoryevich Stolpner, Margarita Iosifovna Levina. Moskau: Mysl.

Gulyga Arseniy Vladimirovich. "Revolyutsiya dukha (Zhizn i tvorchestvo Immanuila Kanta)". In: Kant Immanuil. Sochineniya $v$ vosmi tomakh. Ed. Arseniy Vladimirovich Gulyga. Bd. 1. Moskau: ChORO, 5-49.

${ }^{76}$ Kant, "Brief an die russische Kaiserin Elisabeth vom 14. Dezember 1758”, 6.

${ }^{77}$ Immanuil Kant, "Vseobshchaya estestvennaya istoriya i teoriya neba", in: Immanuil Kant, Sochineniya v vosmi tomakh, ed. Arseniy Vladimirovich Gulyga, Bd. 1 (Moskau: ChORO, 1994), 114. Kant, "Allgemeine Naturgeschichte und Theorie des Himmels", 219. 
Immanuelis Kantii opera ad philosophiam criticam. 1796-1798. Transl. Friedrich Gottlov Born. Vol. I-IV. Leipzig: Schwickert.

Immanuil Kant. Bibliograficheskiy ukazatel literatury na russkom yazyke 1803-1994 gg. 1996. Ed. Lyudmila Sergeyevna Davydova. Moskau: IFRAN.

Kant Immanuel. 1910. "Allgemeine Naturgeschichte und Theorie des Himmels". In: Kant's gesammelte Schriften. Ed. Königlich Preußischen Akademie der Wissenschaften. Bd. I. Berlin: Georg Reimer, 215-368.

Kant Immanuel. 1922. "Brief an die russische Kaiserin Elisabeth vom 14. Dezember 1758”. In: Kant's gesammelte Schriften. Ed. Königlich Preußischen Akademie der Wissenschaften. Bd. X. Berlin: Georg Reimer, 5-6.

Kant Immanuel. 1922. "Brief an Friedrich II. vom 19. März 1770". In: Kant's gesammelte Schriften. Hrsg. von der Königlich Preußischen Akademie der Wissenschaften. Bd. X. Berlin: Georg Reimer, 92-93.

Kant Immanuel. 1922. "Brief an Friedrich II. vom 8. April 1756". In: Kant's gesammelte Schriften. Hrsg. von der Königlich Preußischen Akademie der Wissenschaften. Bd. X. Berlin: Georg Reimer, 3.

Kant Immanuel. 1997. "Osnovopolozheniye k metafizike nravov". Transl. Andrey Konstantinovich Sudakov. In: Kant Immanuil. Sochineniya na nemetskom i russkom yazykakh. Ed. Nelli Vasilyevna Motroshilova, Burkhard Tuschling. Bd. 3. Moskau: Moskovskiy filosofskiy fond, 39-276.

Kant Immanuel. 1914. "Prolegomena zu einer jeden künftigen Metaphysik, die als Wissenschaft wird auftreten können". In: Kant's gesammelte Schriften. Ed. Königlich Preußischen Akademie der Wissenschaften. Bd. IV. Berlin: Georg Reimer, 255-383.

Kant Immanuel. 1994. "Religiya v predelakh tolko razuma". In: Kant Immanuil. Sochineniya $v$ vosmi tomakh. Ed. Arseniy Vladimirovich Gulyga. Bd. 6. Moskau: ChORO, 5-223.

Kant Immanuel. 1838-1842. Sämmtliche Werke. Ed. Karl Rosenkranz, Friedrich Wilhelm Schubert. Bd. 1-12. Leipzig: Leopold Voss.

Kant Immanuil. 2016. "Estestvennoye pravo Feyerabenda". Transl. Lyudmila Eduardovna Kryshtop. Kantovskiy sbornik 35, 3: 75-81; 35, 4: 55-62.

Kant Immanuil. 1964. "Kritika chistogo razuma". Transl. Nikolay Onufriyevich Losskiy, Tsolak Gevorkovich Arzakanyan, Moisey Isaakovich Itkin. In: Kant Immanuil. Sochineniya $v$ shesti tomakh. Ed. Valentin Ferdinandovich Asmus, Arseniy Vladimirovich Gulyga, Teodor Ilich Oyzerman. Bd. 3. Moskau: Mysl, 69-756.

Kant Immanuil. 1994. "Kritika chistogo razuma". Transl. Nikolay Onufriyevich Losskiy, Tsolak Gevorkovich Arzakanyan, Moisey Isaakovich Itkin, Iskra Stepanovna Adreeva. In: Kant Immanuil. Sochineniya v vosmi tomakh. Ed. Arseniy Vladimirovich Gulyga. Bd. 3. Moskau: ChORO, 5-678. 
Kant Immanuil. 2006. "Kritika chistogo razuma”. Transl. Nikolay Onufriyevich Losskiy, Tsolak Gevorkovich Arzakanyan, Moisey Isaakovich Itkin, Nelli Vasilyevna Motroshilova. In: Kant Immanuil. Sochineniya na nemetskom i russkom yazykakh. Ed. Nelli Vasilyevna Motroshilova, Burkhard Tuschling. Bd. 2.1. Moskau: Nauka, 5-1071.

Kant Immanuil. 2006. "Kritika chistogo razuma”. Transl. Nikolay Onufriyevich Losskiy, Tsolak Gevorkovich Arzakanyan, Moisey Isaakovich Itkin, Nelli Vasilyevna Motroshilova. In: Kant Immanuil. Sochineniya na nemetskom i russkom yazykakh. Ed. Nelli Vasilyevna Motroshilova, Burkhard Tuschling. Bd. 2.2. Moskau: Nauka, 9-505.

Kant Immanuil. 1965. "Ob iznachalno zlom v chelovecheskoy prirode". In: Kant Immanuil. Sochineniya $v$ shesti tomakh. Bd. 4(2). Ed. Valentin Ferdinandovich Asmus. Moskau: Mysl, 5-58.

Kant Immanuil. 1965. “Osnovy metafiziki nravstvennosti”. In: Kant Immanuil. Sochineniya $v$ shesti tomakh. Ed. Valentin Ferdinandovich Asmus, Arseniy Vladimirovich Gulyga, Teodor Ilich Oyzerman. Bd. 4(1). Ed. Valentin Ferdinandovich Asmus. Moskau: Mysl.

Kant Immanuil. 1965. "Osnovy metafiziki nravstvennosti". In: Sochineniya $v$ shesti tomakh. Ed. Valentin Ferdinandovich Asmus, Arseniy Vladimirovich Gulyga, Teodor Ilich Oyzerman. Bd. 4(1). Ed. Valentin Ferdinandovich Asmus. Moskau: Mysl, 219-310.

Kant Immanuil. 1980. "Pismo k imperatritse Elizavete Petrovne ot 14 dekabrya 1758 g.. In: Kant Immanuil. Traktaty i pisma. Ed. Arseniy Vladimirovich Gulyga. Moskau: Nauka, 505.

Kant Immanuil. 1994. "Pismo k imperatritse Elizavete Petrovne ot 14 dekabrya 1758 g.. In: Kant Immanuil. Sochineniya v vosmi tomakh. Ed. Arseniy Vladimirovich Gulyga. Bd. 8. Moskau: ChORO, 464-465.

Kant Immanuil. 1994. "Prolegomeny ko vsyakoy budushchey metafizike, kotoraya mozhet poyavitsya kak nauka". Transl. Vladimir Sergeyevich Solovyev, Boris Aleksandrovich Fokht, Mikhail Aleksandrovich Dynnik, Moisey Isaakovich Itkin, M. M. Belyaev. In: Kant Immanuil. Sochineniya $v$ vosmi tomakh. Ed. Arseniy Vladimirovich Gulyga. Bd. 4. Moskau: ChORO, 5-152.

Kant Immanuil. 1965. "Prolegomeny ko vsyakoy budushchey metafizike, mogushchey poyavitsya kak nauka”. Transl. Vladimir Sergeyevich Solovyev, Boris Aleksandrovich Fokht, Mikhail Aleksandrovich Dynnik, Moisey Isaakovich Itkin. In: Kant Immanuil. Sochineniya $v$ shesti tomakh. Ed. Valentin Ferdinandovich Asmus, Arseniy Vladimirovich Gulyga, Teodor Ilich Oyzerman. Bd. 4(1). Moskau: Mysl, 67-210. 
Kant Immanuil. 1994. "Vseobshchaya estestvennaya istoriya i teoriya neba". In: Kant Immanuil. Sochineniya $v$ vosmi tomakh. Ed. Arseniy Vladimirovich Gulyga. Bd. 1. Moskau: ChORO, 113-260.

Kant Immanuil. 2000. Iz rukopisnogo naslediya (materialy $k$ „Kritike chistogo razuma", Opus postumum). Ed. Vladimir Aleksandrovich Zhuchkov. Moskau: Nauka.

Kant Immanuil. 1896-1897. Kritika chistago razuma. Transl. Nikita Matveyevich Sokolov. Sankt-Petersburg: Popov.

Kant Immanuil. 1902. Kritika chistago razuma. Transl. Nikita Matveyevich Sokolov. Sankt-Petersburg: Popov.

Kant Immanuil. 1907. Kritika chistago razuma. Transl. Nikolay Onufriyevich Losskiy. Sankt-Peterburg: Tipografiya Stasyulevicha.

Kant Immanuil. 1915. Kritika chistago. Transl. Nikolay Onufriyevich Losskiy. Petrograd: Tipografiya Stasyulevicha.

Kant Immanuil. 1867. Kritika chistago razuma. Transl. Mikhail Ivanovich Vladislavlev. Sankt-Petersburg: Tipografiya N. Tiblena.

Kant Immanuil. 1994. Kritika chistogo razuma. Transl. Nikolay Onufriyevich Losskiy, Tsolak Gevorkovich Arzakanyan, Moisey Isaakovich Itkin. Moskau: Mysl.

Kant Immanuil. 1998. Kritika chistogo razuma. Transl. Nikolay Onufriyevich Losskiy, mit den Varianten der Übersetzung ins Russische und in die anderen europäischen Sprachen. Ed. Vladimir Aleksandrovich Zhuchkov. Moskau: Nauka.

Kant Immanuil. 2016. Lektsii of filosofskom uchenii o religii (redaktsiya K. G. L. Pelitsa). Transl. Lyudmila Eduardovna Kryshtop. Ed. Aleksey Nikolayevich Kruglov. Moskau: Kanon.

Kant Immanuil. 2000. Lektsii po etike. Ed. Abdusalam Abdulkerimovich Guseynov. Moskau: Respublika.

Kant Immanuil. 1912. Osnovopolozheniye $k$ metafizike nravov. Transl. L. D. B. Ed. Veniamin Mikhaylovich Khvostov. Moskau: Tipografiya Vilde.

Kant Immanuil. 1937. Prolegomeny ko vsyakoy budushchey metafizike, mogushchey vozniknut v kachestve nauki. Transl. Vladimir Sergeyevich Solovyev, Boris Aleksandrovich Fokht, Mikhail Aleksandrovich Dynnik. Moskau: Sotsekgiz.

Kant Immanuil. 1889. Prolegomeny ko vsyakoy budushchey metafizike, mogushchey vozniknut v smysle nauki. Transl. Vladimir Sergeyevich Solovyev. Sankt-Petersburg: Tipografiya Gattsuka.

Kant Immanuil. 1934. Prolegomeny. Transl. Vladimir Sergeyevich Solovyev, Boris Aleksandrovich Fokht. Ed. Artashes Khorenovich Saradzhev. Moskau: OGIZ.

Kant Immanuil. 1908. Religiya i predelakh tolko razuma. Transl. Nikita Matveyevich Sokolov. Sankt-Peterburg: Izdaniye Yakovenko.

Kant Immanuil. 1940. Sochineniya 1747-1777: v dvukh tomakh. Transl. Boris Aleksandrovich Fokht. Ed. Boris Yulyevich Slivker. Bd. 2: 1759-1777 gg. Moskau: Sotsekgiz. 
Kant Immanuil. 1994-2019. Sochineniya na nemetskom i russkom yazykakh. Ed. Nelli Vasilyevna Motroshilova, Burkhard Tuschling. Bd. 1-5. Moskau: Kami, Nauka, Moskovskiy filosofskiy fond, Kanon.

Kant Immanuil. 1963-1966. Sochineniya $v$ shesti tomakh. Ed. Valentin Ferdinandovich Asmus, Arseniy Vladimirovich Gulyga, Teodor Ilich Oyzerman. Moskau: Mysl.

Kant Immanuil. 1994. Sochineniya v vosmi tomakh. Ed. Arseniy Vladimirovich Gulyga. Moskau: ChORO.

Kantovo osnovaniye dlya metafiziki nravov. 1803. Transl. Yakov Andreyevich Ruban. Nikolayev: V tipografii Chernomorskago shturmanskago uchilishcha.

Krouglov Alexei N. 2008. "Zum Begriff der Kausalität bei I. Kant”. In: Kant im Spiegel der russischen Kantforschung heute. Ed. Norbert Hinske, Nelly Motroschilowa. Stuttgart-Bad Cannstatt: frommann-holzboog, 53-69.

Krouglov Alexei N., Dieter Hüning. 2019. "Neuer Band der deutsch-russischen Kant-Ausgabe erschienen". Kant-Studien 110: 186-188.

Kruglov Aleksey Nikolayevich. 2017. "O nekotorykh istochnikakh i problemakh perevoda kantovskogo termina „Ding an sich“" Istoriko-filosofskiy ezhegodnik 33: 137-164.

Kruglov Aleksey Nikolayevich. 2019. “,Vashego Imper. Velichestva vsepoddanneyshiy rab Immanuil Kant": o nekotorykh trudnostyakh perevoda pisma Kanta k tsaritse Elizavete Petrovne”. In: Transfer - Kultur - Akteur. Sbornik statey k 60-letiyu Dirka Kempera. Ed. Natalia Aleksandrovna Bakshi, Aleksey Iosifovich Zherebin, Ivonne Pertsgen, Elizabet Shore. Moskau: RGGU, 197-203.

Krystop, Ludmila Eduardovna. 2012. "Etika chistogo obraza mysley Kanta". In: Istoriya filosofii i sotsiokulturnyy kontekst. Materialy Mezhdunarodnoy konferentsii. Moskva. 24-25 dekabrya 2012 g. Ed. Taras Aleksandrovich Shiyan. Moskau: RGGU, 158-167.

Mennicke Carl. 1906. Hasse und die Brüder Graun als Symphoniker. Nebst Biographien und thematischen Katalogen. Leipzig: Breitkopf \& Härtel.

Molchanov Viktor Igorevich. 2017. "Bezotnositelnoye i nezavisimoye. O razlichii terminov i slov v „Kritike chistogo razuma“ Kanta”. Istoriko-filosofskiy ezhegodnik 32: 103-133.

Moskovskiye vedomosti. 1893. April, 10, 97: 5.

Motroshilova Nelli Vasilyevna. 2006. "Kommentarii k novoy redaktsii perevoda „Kritiki chistogo razuma". In: Kant Immanuil. Sochineniya na nemetskom i russkom yazykakh. Ed. Nelli Vasilyevna Motroshilova, Burkhard Tuschling. Bd. 2.2. Moskau: Nauka, 699-715.

Motroshilova Nelli Vasilyevna. 1994. "Predislovie". In: Kant Immanuil. Sochineniya na nemetskom i russkom yazykakh. Ed. Nelli Vasilyevna Motroshilova, Burkhard Tuschling. Bd. 1. Moskau: Kami, 42-73. 
Müller Andreas. 1828. Anleitung zum geistlichen Geschäfts-Style und zur geistlichen Geschäfts-Verwaltung sowohl nach der Pastoral und dem gemeinen Kirchenrechte als nach besonderen bayererischen Verordnungen. Nebst einem Anhange von Formularen aller Arten von Geschäfts-Aufsätzen, welche in d. verschiedenen Verzweigungen der geistlichen Amts-Verwaltung vorkommen, zunächst für katholische Geistliche. Würzburg: Etlinger.

Narezhnyy Vasiliy Trofimovich. 1983. Rossiyskiy Zhilblaz, ili Pokhozhdeniya knyazya Gavrily Simonovicha Chistyakova. Roman v shesti chastyakh. Petrozavodsk: Kareliya.

"Ot izdatelstva”. 1963. In: Kant Immanuil. Sochineniya $v$ shesti tomakh. Ed. Valentin Ferdinandovich Asmus, Arseniy Vladimirovich Gulyga, Teodor Ilich Oyzerman. Bd. 1. Ed. Arseniy Vladimirovich Gulyga. Moskau: Mysl, 5-6.

Oyzerman Teodor Ilich. 1963. "Immanuil Kant - rodonachalnik klassicheskoy nemetskoy filosofii". In: Kant Immanuil. Sochineniya $v$ shesti tomakh. Ed. Valentin Ferdinandovich Asmus, Arseniy Vladimirovich Gulyga, Teodor Ilich Oyzerman. Bd. 1. Ed. Arseniy Vladimirovich Gulyga. Moskau: Mysl, 7-49.

Palmquist Stephen R. 2015. "What is Kantian Gesinnung? On the Priority of Volition over Metaphysics and Psychology in Religion within the Bounds of Bare Reason". Kantian Review 20-22: 235-264.

"Perepiska Immanuila Kanta i Ioganna Georga Gamana". 2010. Transl. Vladimir Khamitovich Gilmanov. Kantovskiy sbornik 29, 1: 90-111.

Pinheiro Leticia Machado. 2005. "O conceito kantiano de intenção 'Gesinnung' em "Sobre o mal radical na natureza humana"'. Revista Portuguesa de Filosofia 61: 1019-1026.

Slivker Boris Yulyevich. 1933. “„Dokriticheskiy“ Kant”. Pod znamenem marksizma 4: 179-214.

Syn Otechestva. 1893. 12. (24.) April, 98: 2.

Wagner Günter. 1994. Die Sinfonien Carl Philipp Emanuel Bachs: werdende Gattung und Originalgenie. Stuttgart: Metzler.

Warda Arthur. 1919. Die Druckschriften Immanuel Kants (bis zum Jahre 1838). Wiesbaden: Staadt.

Zur Erinnerung an Gotthold Ephraim Lessing. Briefe und Aktenstücke aus den Papieren der Herzoglichen Bibliothek und den Akten des Herzoglichen Landeshauptarchivs zu Wolfenbüttel. 1870. Ed. Otto von Heinemann. Leipzig: Hirzel. 


\section{Abstract \\ Russian Editions and Translations of Kant: Preliminary Premises Concerning the Russian-German Edition of Kant (1994-2019)}

The paper introduces preliminary premises concerning editions and translations of Kant in Russian. Some current problems that Russian Kant scholars have faced in recent years working on these editions are presented. Three cases illustrate crucial difficulties within translations and interpretations of Kant, as well as their origin. The first case concerns Kant's expression 'Kausalität der Ursache' in the Critique of pure reason and Prolegomena; the second one involves the term 'Gesinnung' in Kant's critical writings, and finally the third analyzes Kant's letter from 1758 to Tsarina Elisabeth. Although the final volume of new bilingual edition of Kant's key works (1994-2019) has been just published, I conclude that Russian Kant Scholarship is already in need of a brand new edition.

Keywords: Russian Kant Scholarship, Kant's Russian Editions, Kant's Russian Translations, 'Kausalität der Ursache,' 'Gesinnung, Tsarina Elisabeth 